Supporting Information:

\title{
Structure Elucidation and Relative Toxicity of (24R)-24-Hydroxyyessotoxin from a Namibian Isolate of Gonyaulax spinifera
}

Isabelle Rajotte $^{\dagger}$, Cheryl Rafuse ${ }^{\dagger}$, Elliott J. Wright ${ }^{\dagger}$, John C. Achenbach ${ }^{\ddagger}$, Lee D. Ellis ${ }^{\ddagger}$, Pearse $\operatorname{McCarron}^{\dagger}, *$

${ }^{\dagger}$ Biotoxin Metrology, National Research Council Canada, 1411 Oxford Street, Halifax, Nova Scotia B3H 3Z1, Canada

$\$$ Aquatic and Crop Resource Development, National Research Council Canada, 1411 Oxford Street, Halifax, Nova Scotia B3H 3Z1, Canada

*Corresponding author. Tel: + 1 9024266182. Email: pearse.mccarron@,nrc-cnrc.gc.ca 


\section{LIST OF FIGURES}

Figure S1. LC-MS/MS chromatogram showing the YTX profile in Namibian

Gonyaulax spinifera extract with the 45-hydroxyYTX transition from analysis of CRM-FDMT1 overlaid (red).

Figure S2. Comparison of the COSY (a) and TOCSY (b) spectra for 24-hydroxyYTX (7) (red) and YTX (1) (blue) between 3.6 and 3.4 ppm.

Figure S3. (a) ROESY spectrum of $7\left(700 \mathrm{MHz}, \mathrm{CD}_{3} \mathrm{OD}\right)$ between 3.6 and $3.4 \mathrm{ppm}$.

(b) 3D representation of 24-hydroxyYTX (7) and observed ROESY correlations in red.

Figure S4. LC-MS/MS chromatograms showing extracted traces of hydroxyYTX (left) and YTX (right) from reference materials (top) and an Italian Protoceratium reticulatum extract (bottom) (LC-MS method A).

Figure S5. Representative phenotypes of zebrafish larvae exposed to dilution of either YTX (1) (left column) or 24-hydroxyYTX (7) (right column) from 6-120 hours post fertilization (hpf). Carrier control was $0.5 \%$ methanol in HE3 exposure medium. Concentrations of toxin are indicated. Scale bar $=1000 \mu \mathrm{m}$. Yolk edema is indicated by arrow and pericardial edema is indicated by an asterisk.

Figure S6. Full suite of NMR experiments for 24-hydroxyYTX (7) (700 MHz, $\left.\mathrm{CD}_{3} \mathrm{OD}\right)$.

(a) Structure of 24-hydroxyYTX (7); (b) ${ }^{1} \mathrm{H}$; (c) ${ }^{13} \mathrm{C}$; (d) COSY; (e) TOCSY; (f) HSQC; (g) HMBC; (h) ROESY

Figure S7. NMR experiments for YTX (1) (700 MHz, CD $\left.{ }_{3} \mathrm{OD}\right)$.

(a) Structure of YTX (1); (b) ${ }^{1} \mathrm{H}$; (c) ${ }^{13} \mathrm{C}$

Figure S8. Observed LTQ MS fragmentation pathways of YTX (1) and 24hydroxyYTX (7).

Table 1. NMR Spectroscopic Data $\left({ }^{1} \mathrm{H} 700 \mathrm{MHz},{ }^{13} \mathrm{C} 175 \mathrm{MHz}, \mathrm{CD}_{3} \mathrm{OD}\right)$ for 24HydroxyYTX (7) and Yessotoxin (1) 


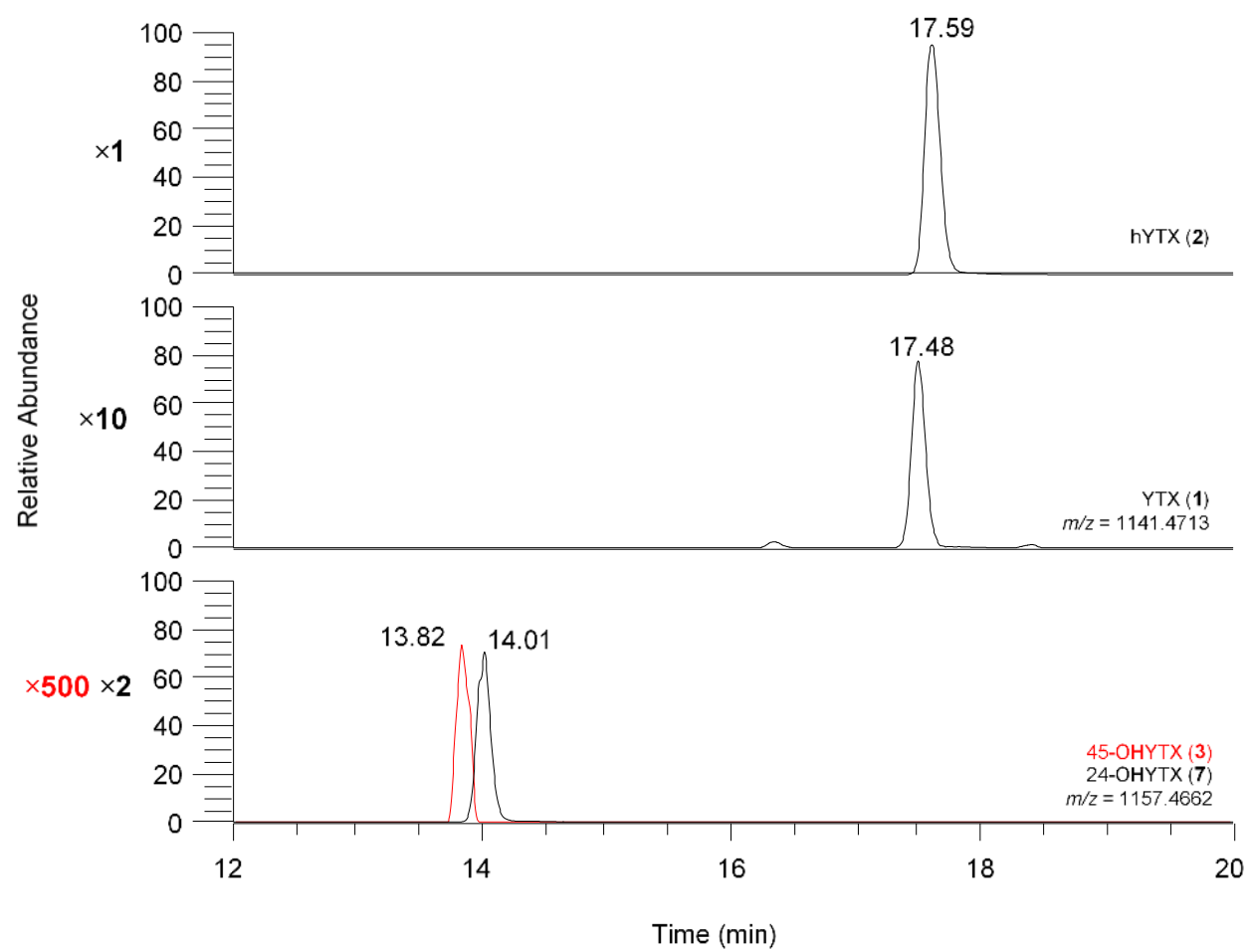

Figure S1. LC-MS/MS chromatogram showing the YTX profile in Namibian Gonyaulax spinifera extract with the 45-hydroxyYTX transition from analysis of CRM-FDMT1 overlaid (red). 
(a)
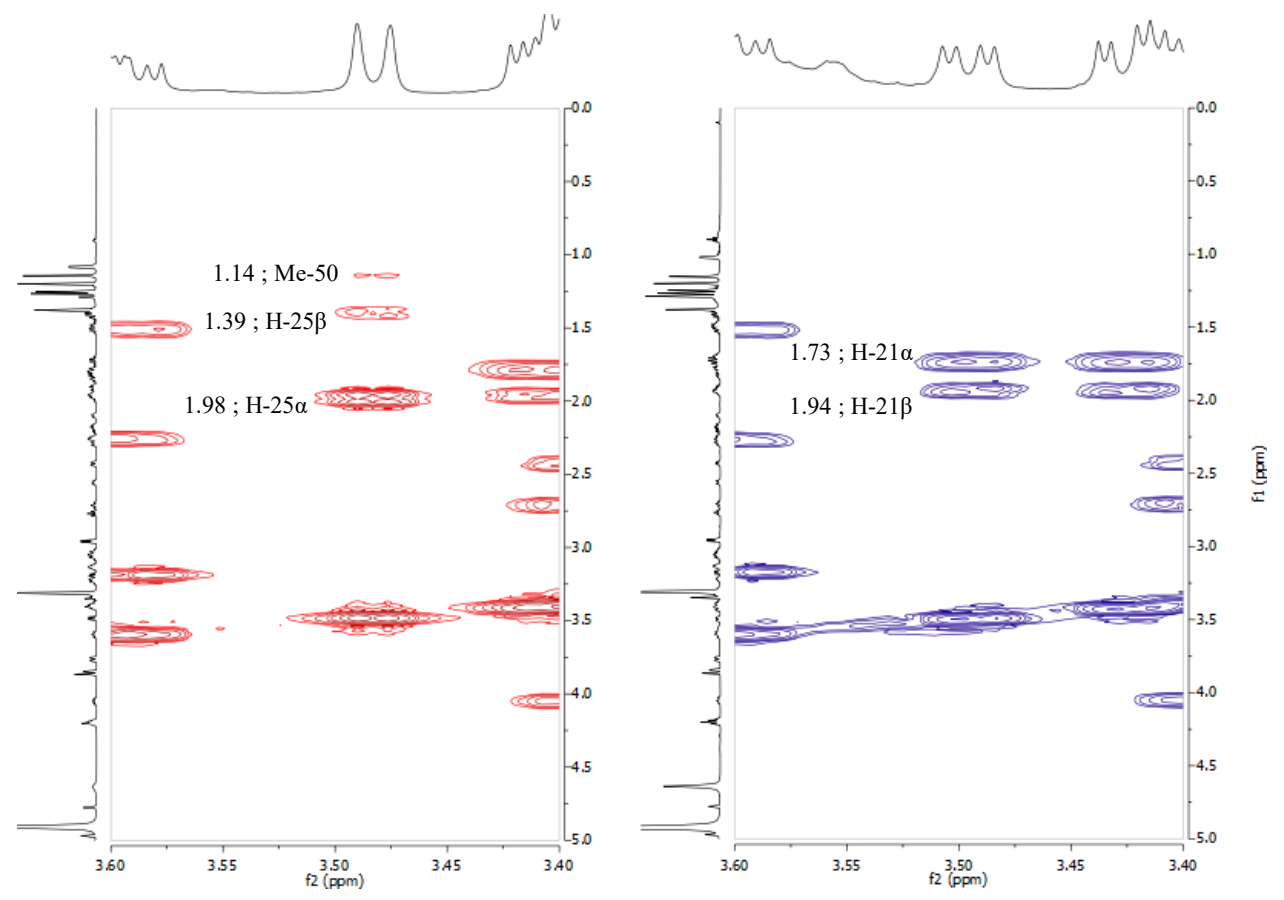

(b)
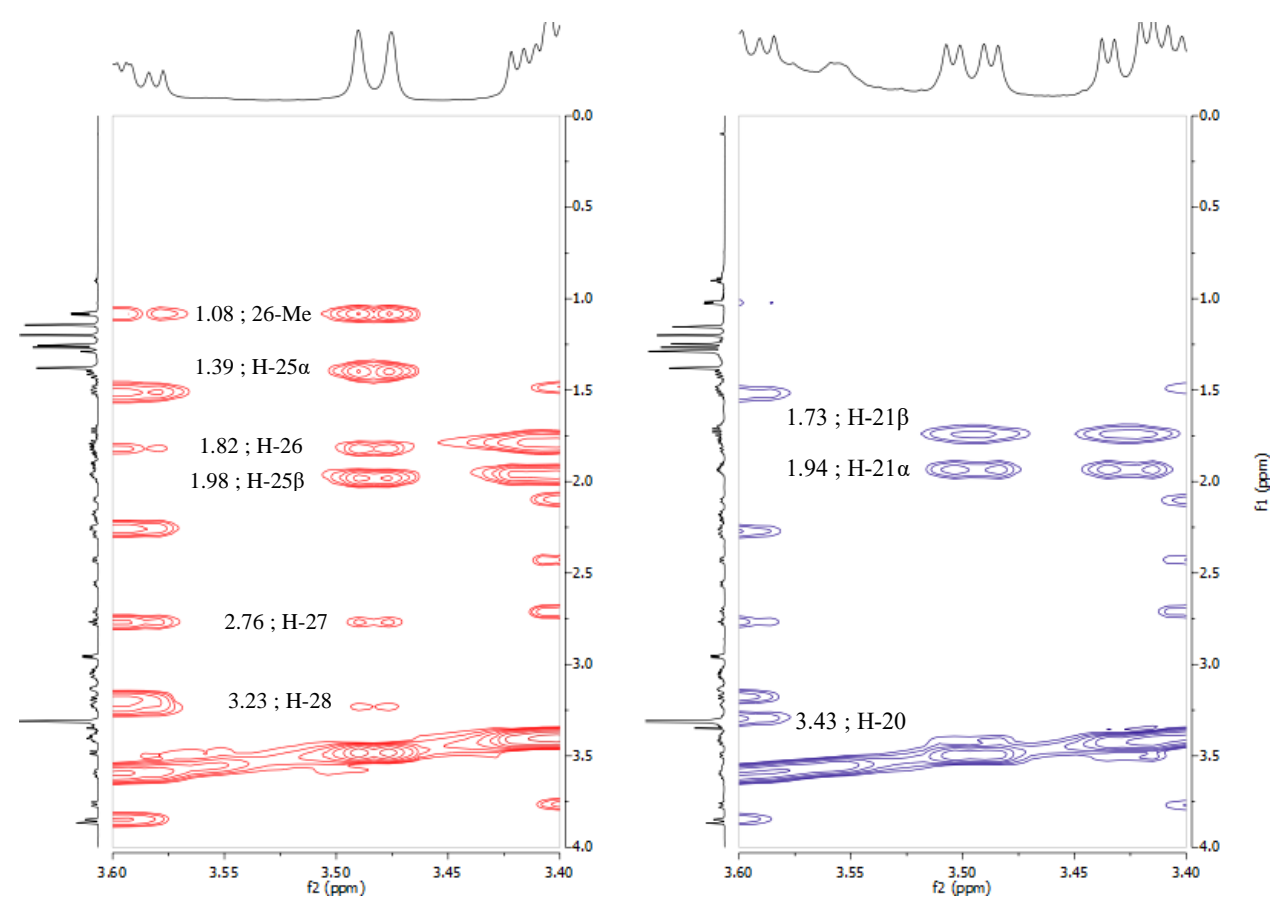

Figure S2. Comparison of the COSY (a) and TOCSY (b) spectra for 24-hydroxyYTX (7) (red) and YTX (1) (blue) between 3.6 and 3.4 ppm. 
(a)
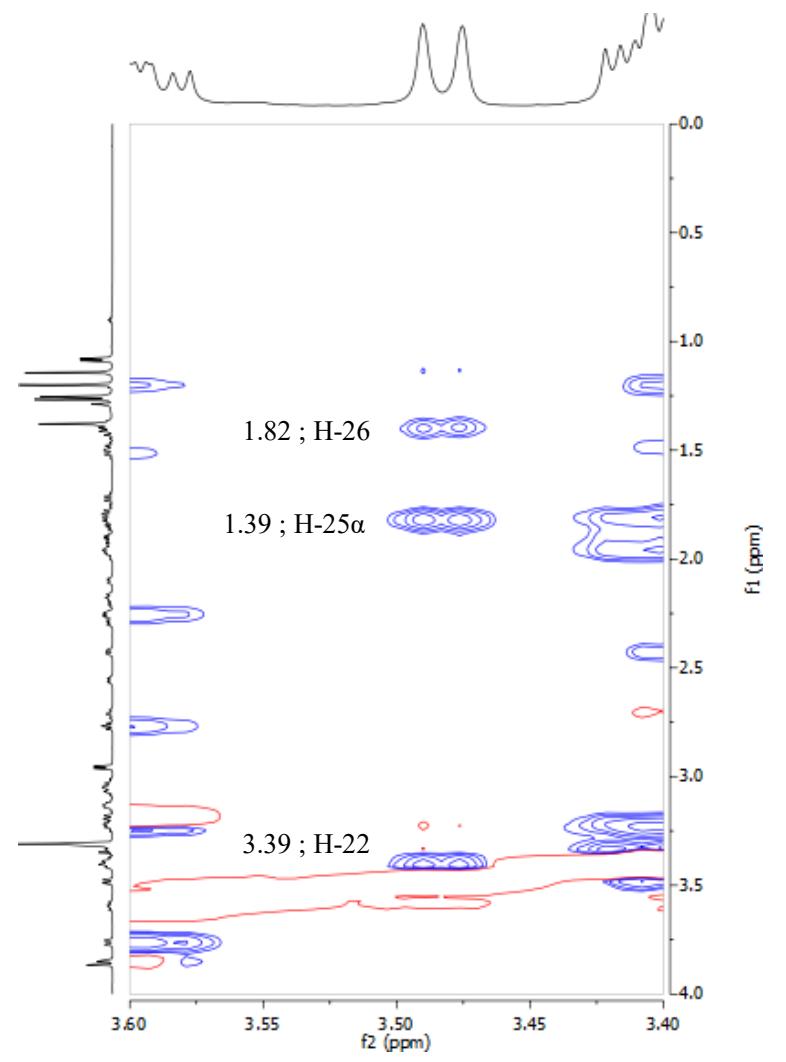

(b)

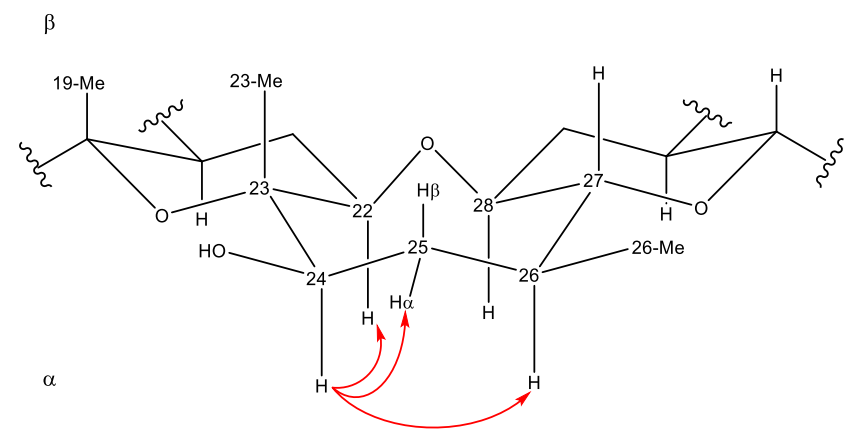

Figure S3. (a) ROESY spectrum of 7 between 3.6 and 3.4 ppm. (b) 3D representation of 24-hydroxyYTX (7) and observed ROESY correlations in red. 


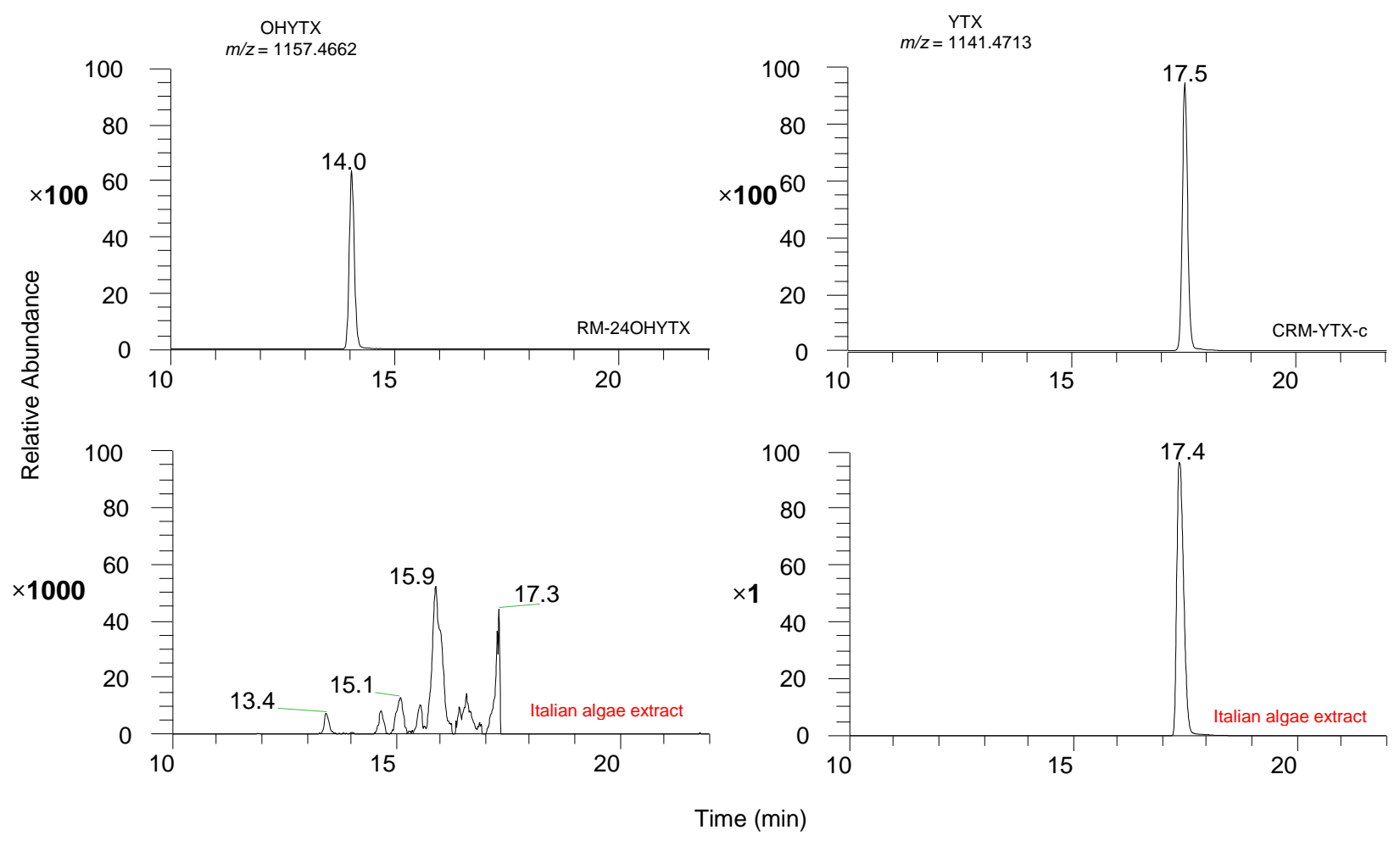

Figure S4. LC-MS/MS chromatograms showing extracted traces of hydroxyYTX (left) and YTX (right) from reference materials (top) and an Italian Protoceratium reticulatum extract (bottom) (LC-MS method A). 
YTX
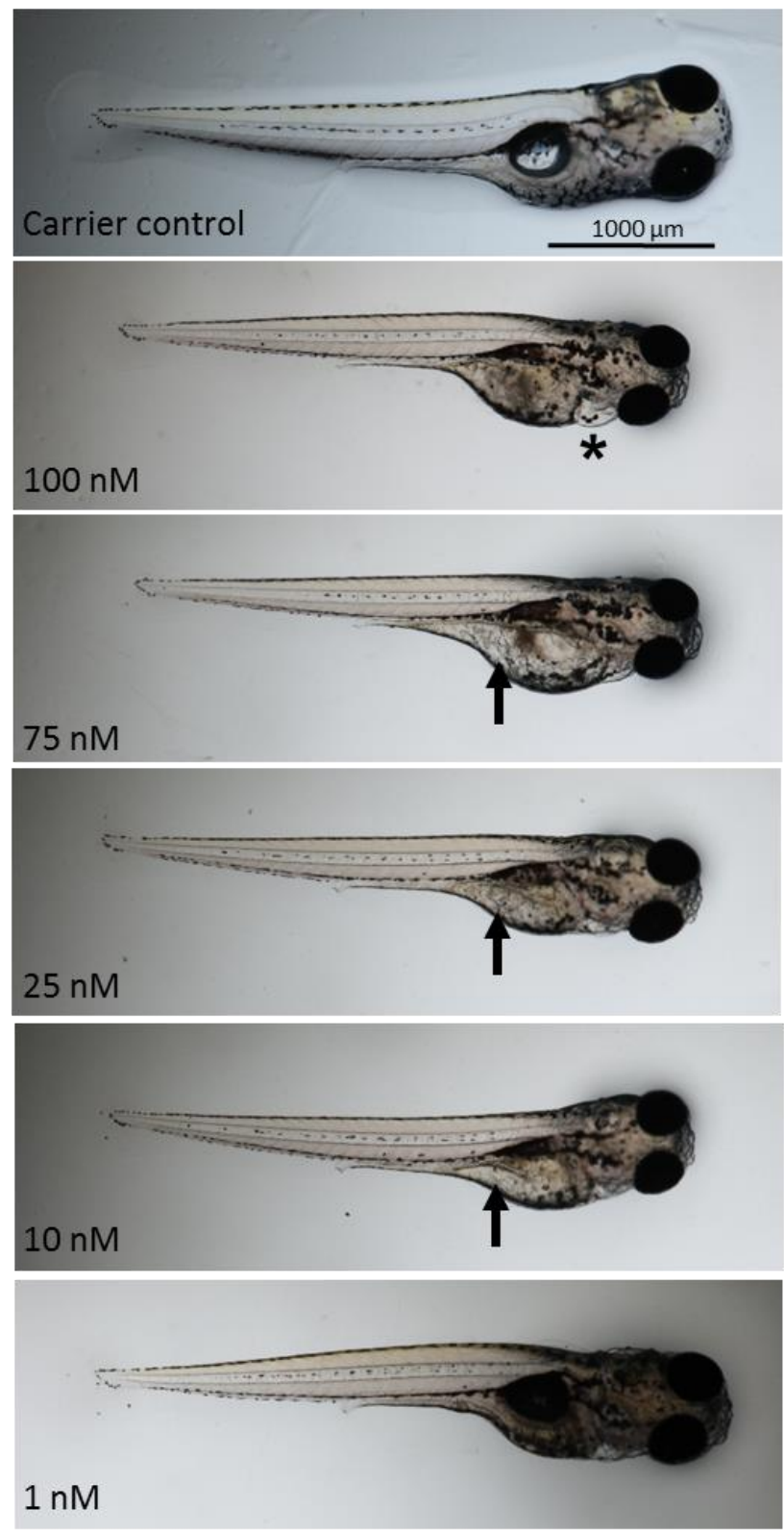

\section{4-OH YTX}

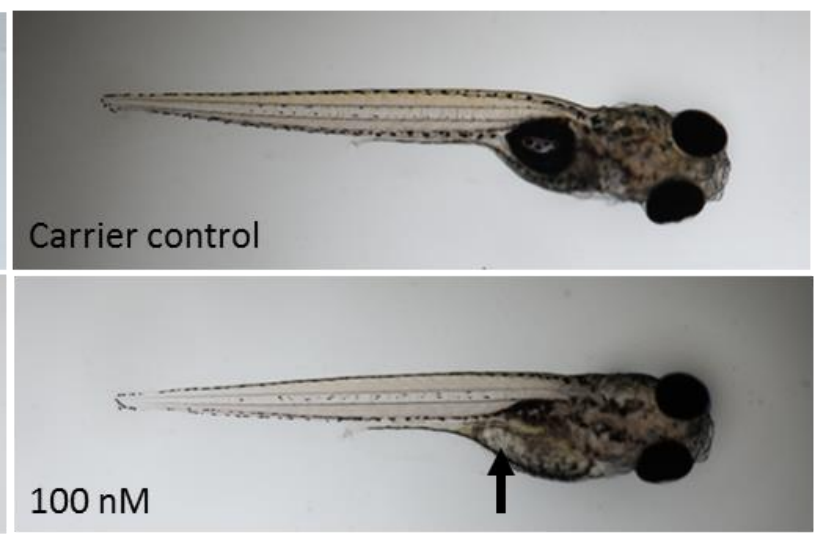

$75 \mathrm{nM}$

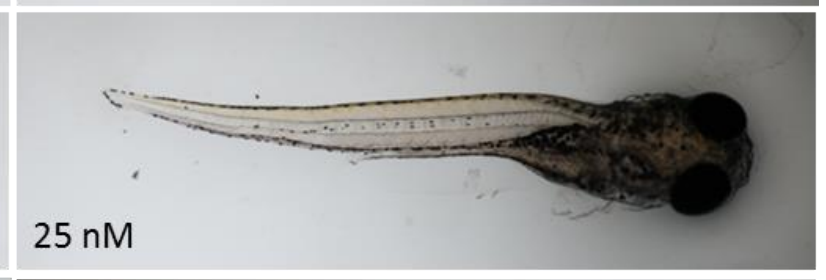

$10 \mathrm{nM}$

Figure S5. Representative phenotypes of zebrafish larvae exposed to dilution of either YTX (1) (left column) or 24-hydroxyYTX (7) (right column) from 6-120 hours post fertilization (hpf). Carrier control was $0.5 \%$ methanol in HE3 exposure medium. Concentrations of toxin are indicated. Scale bar $=1000 \mu \mathrm{m}$. Yolk edema is indicated by arrow and pericardial edema is indicated by an asterisk. 


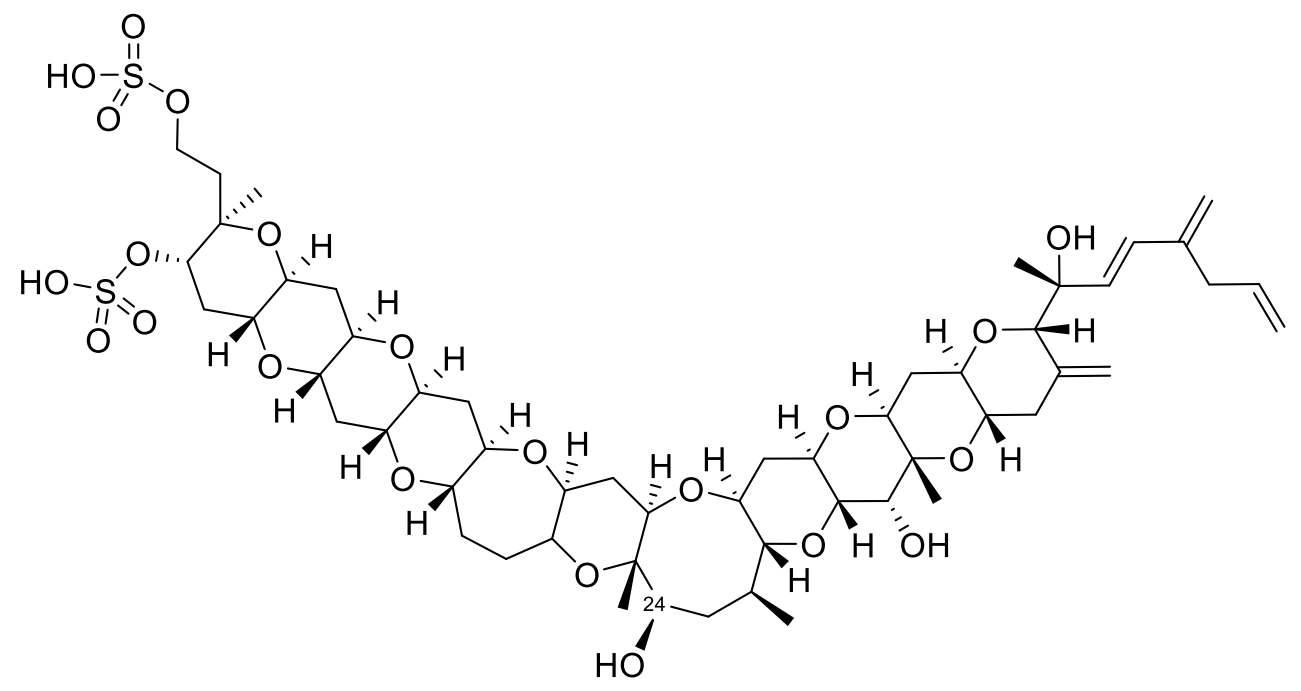

Figure S6. (a) Structure of 24-hydroxyYTX (7) 


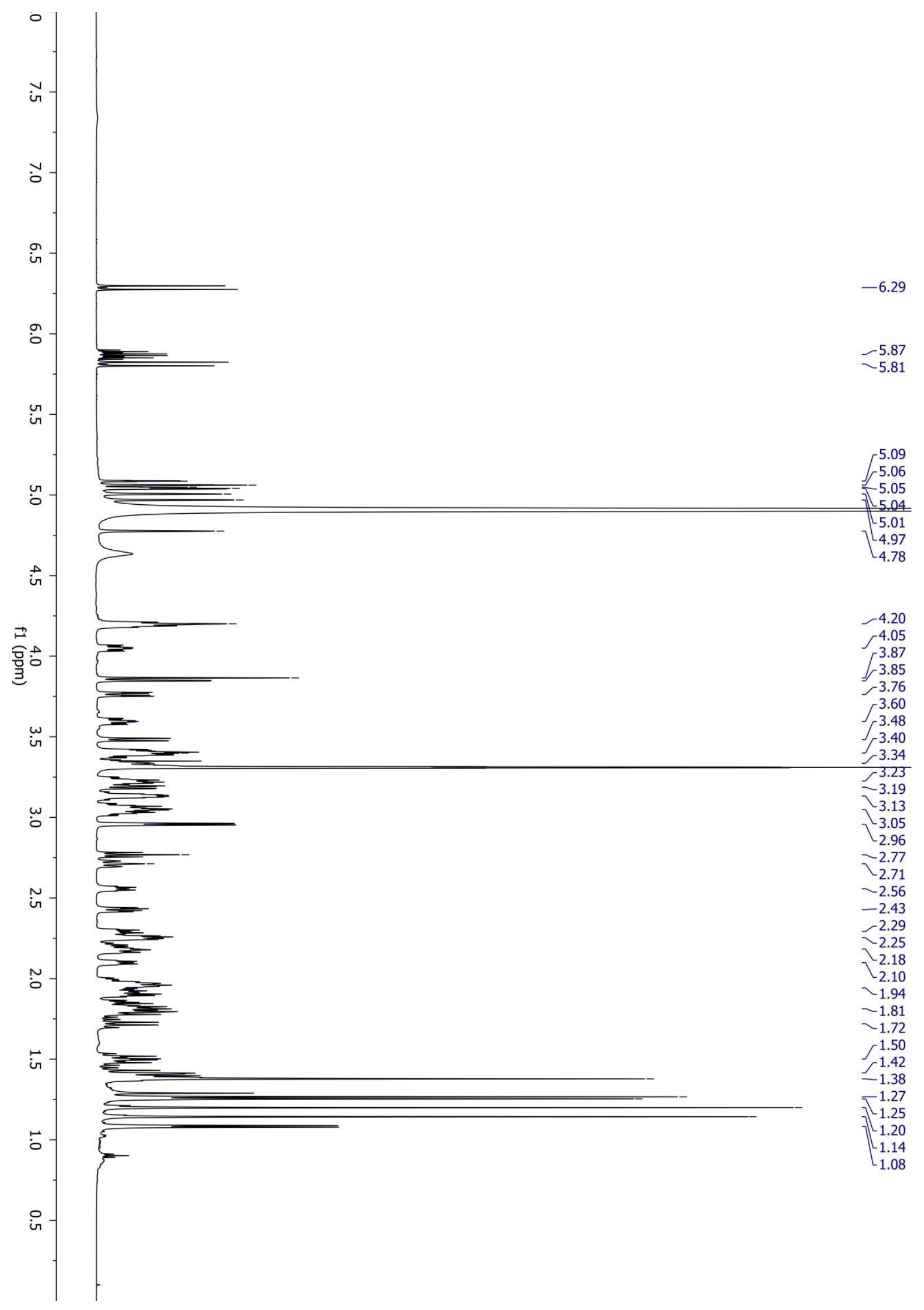

Figure S6. (b) ${ }^{1} \mathrm{H}$ 


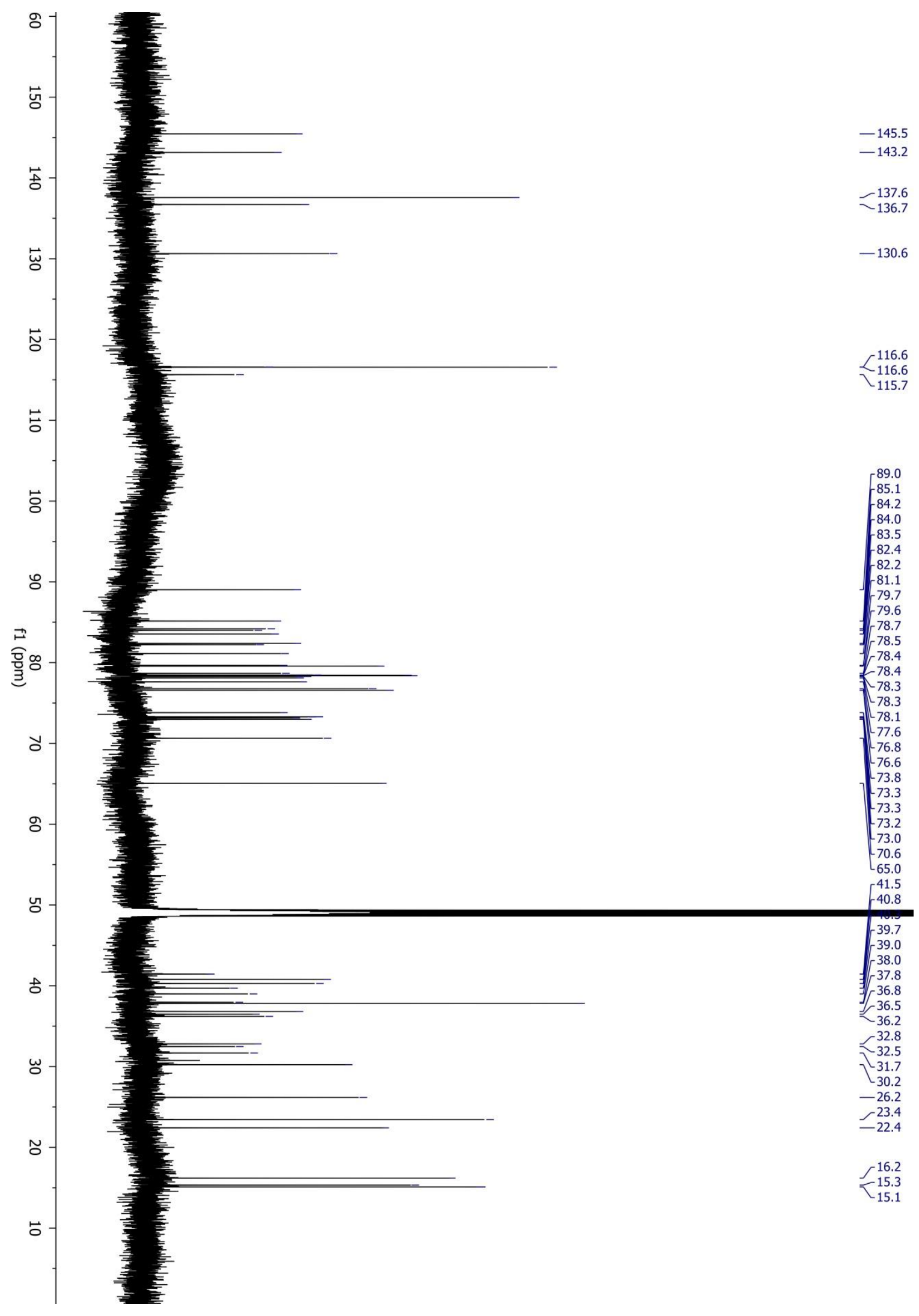

Figure S6. (c) ${ }^{13} \mathrm{C}$ 


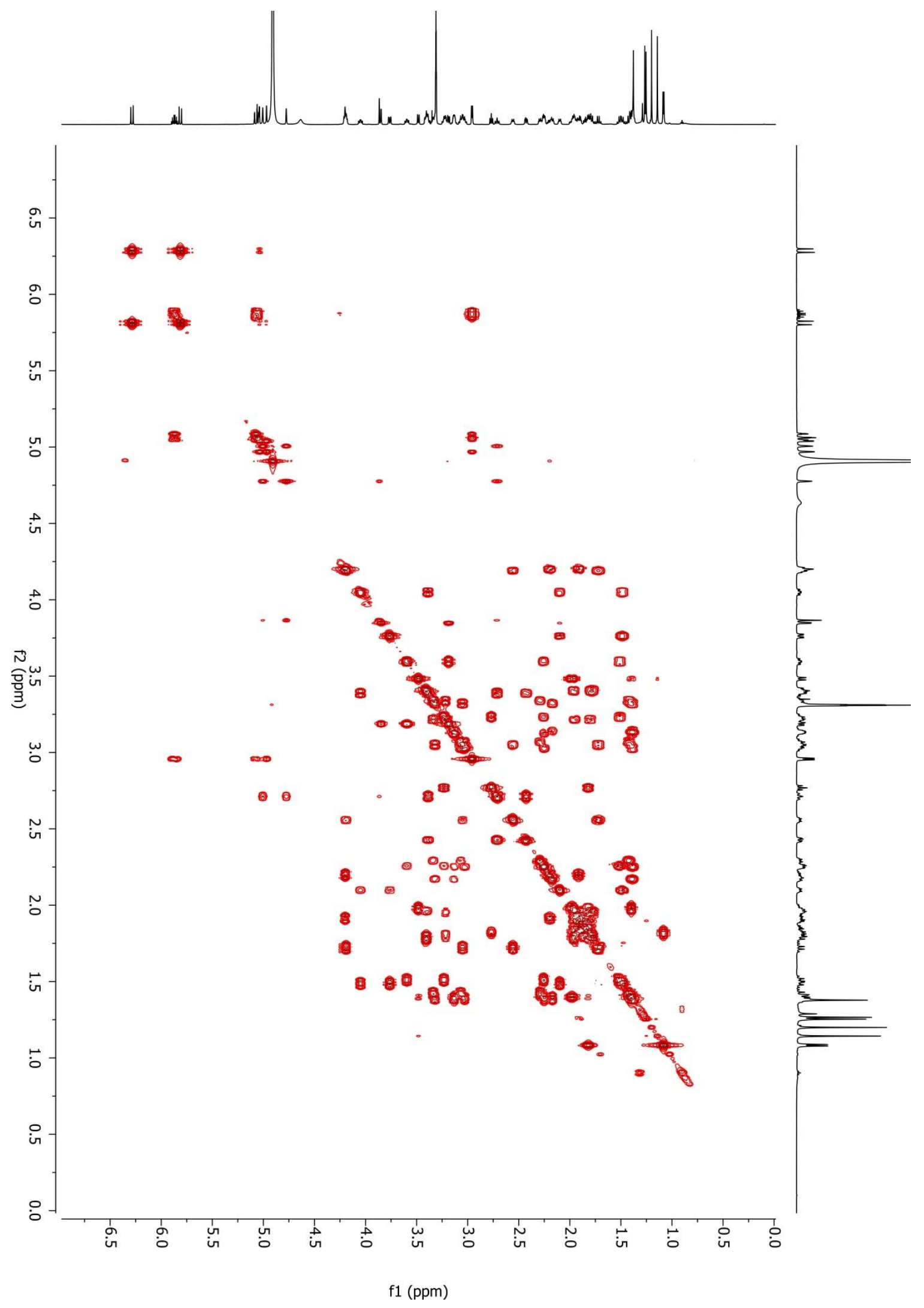

Figure S6. (d) COSY 


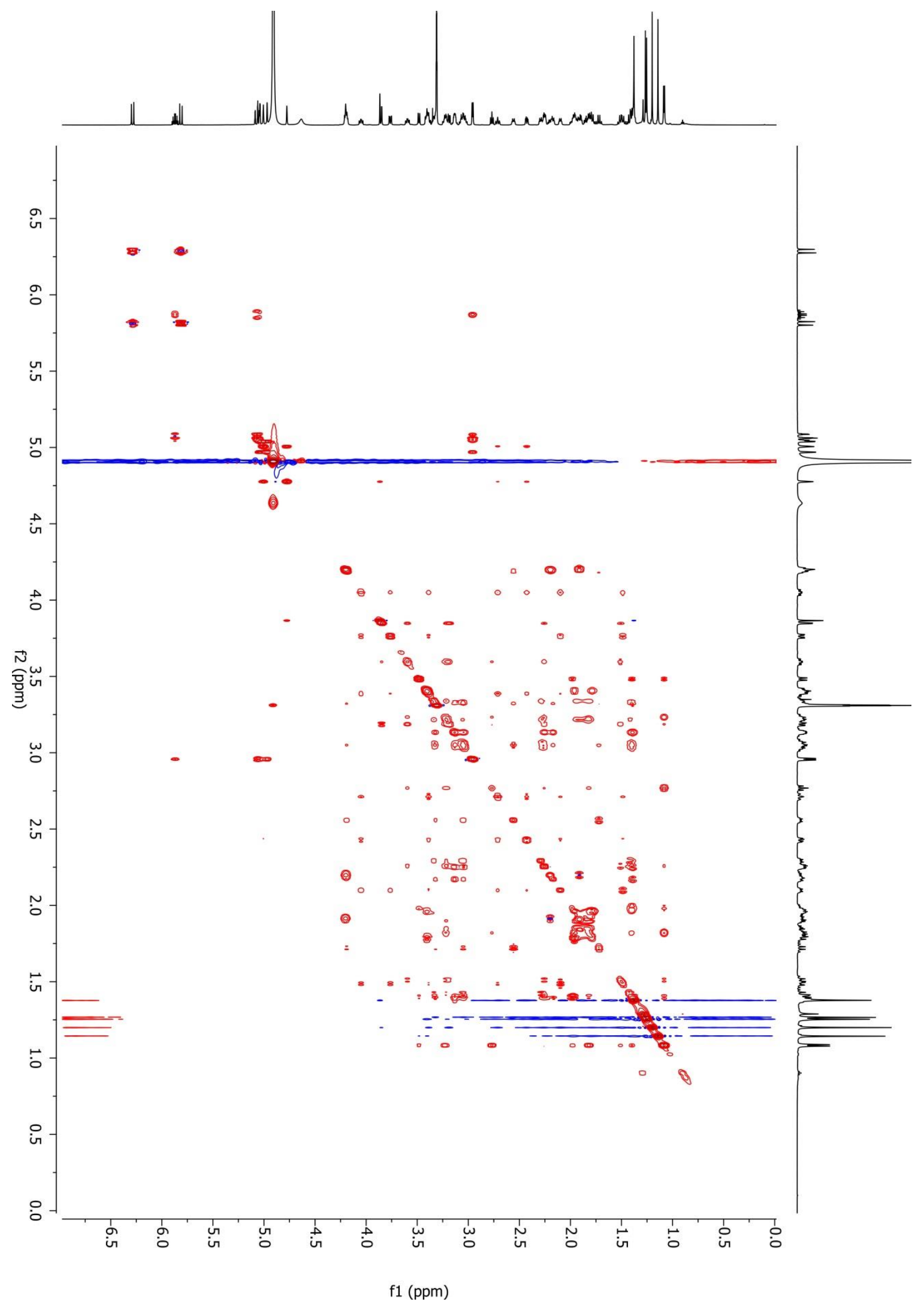

Figure S6. (e) TOCSY 


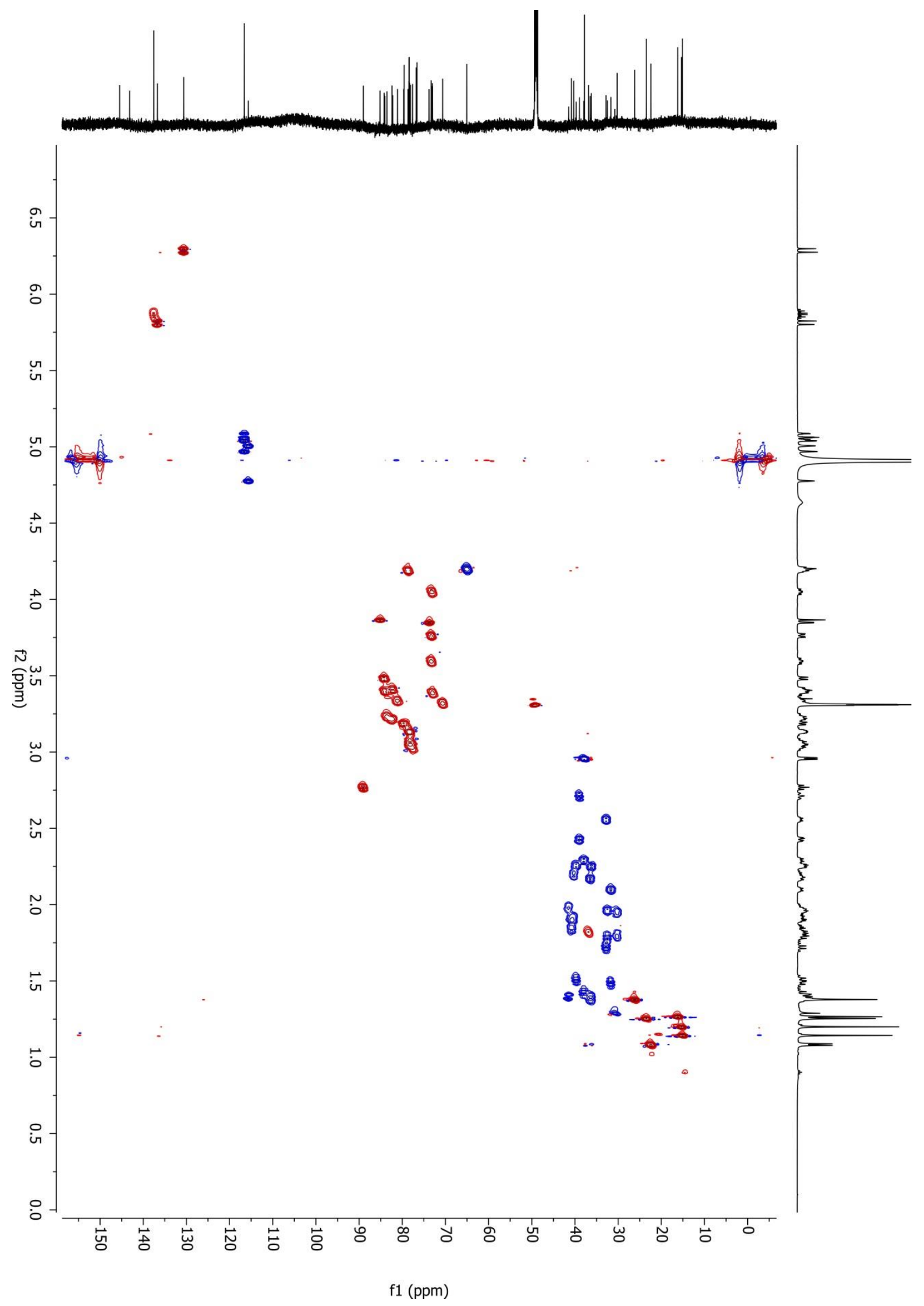

Figure S6. (f) HSQC 


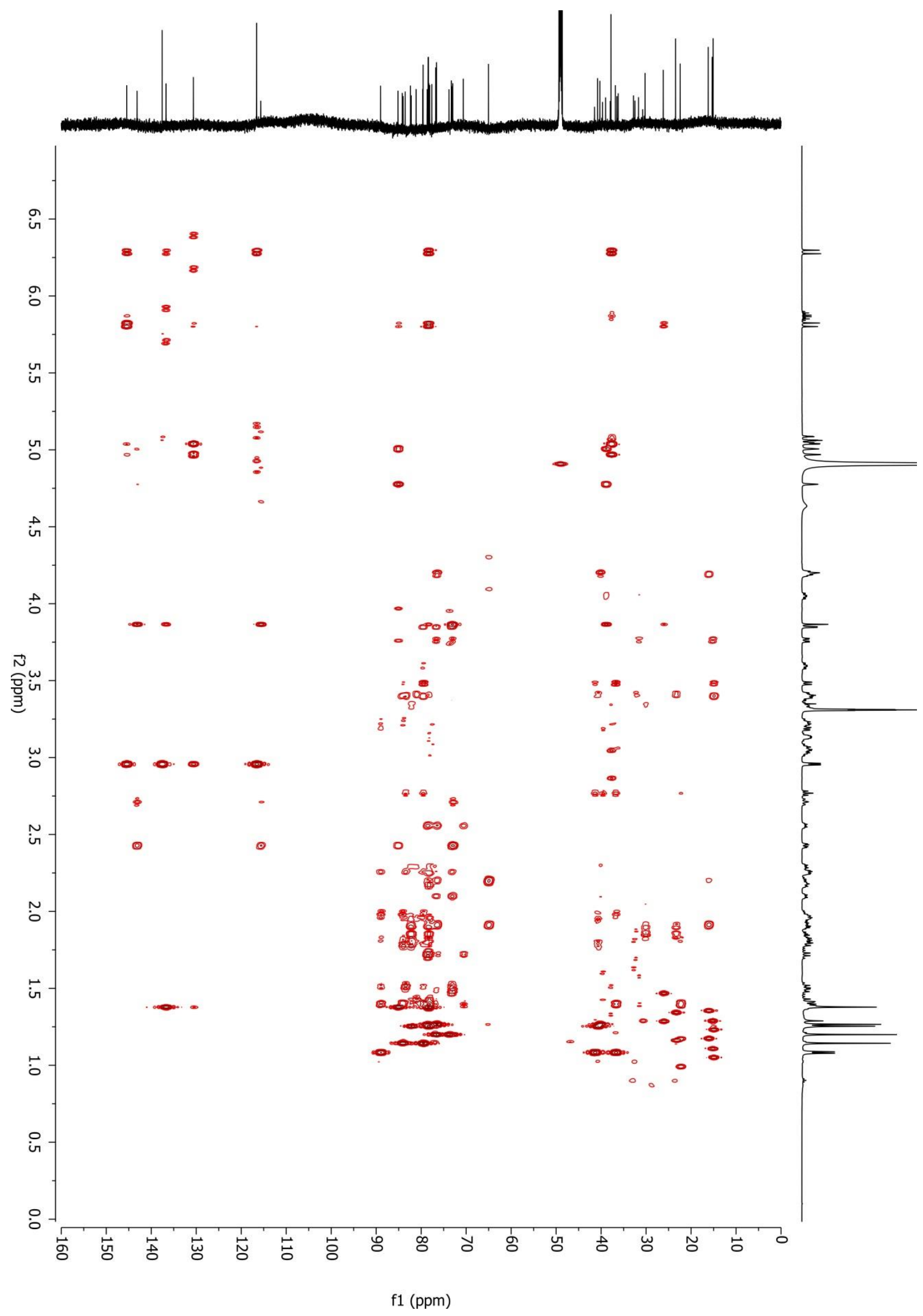

Figure S6. (g) HMBC 


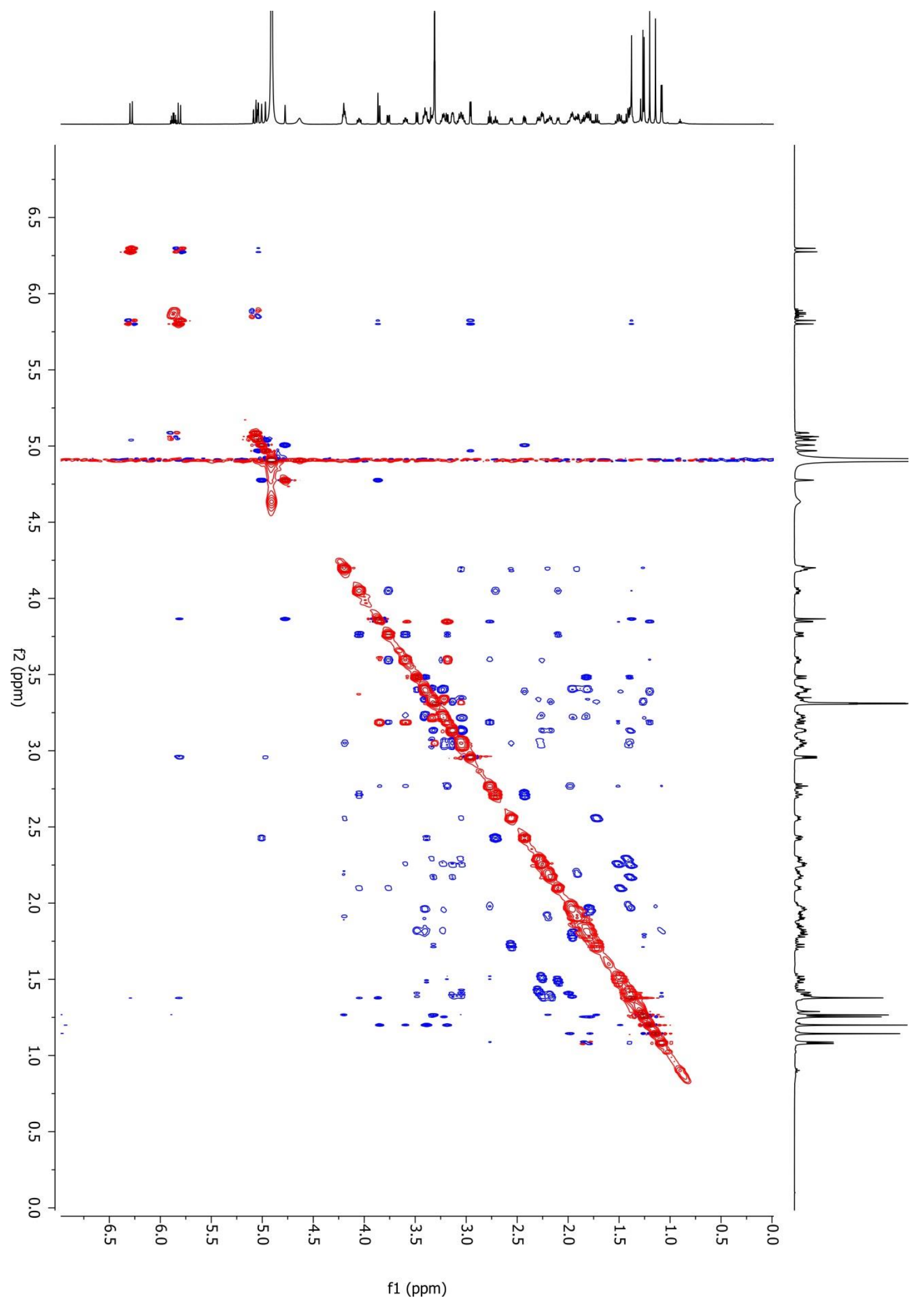

Figure S6. (h) ROESY 


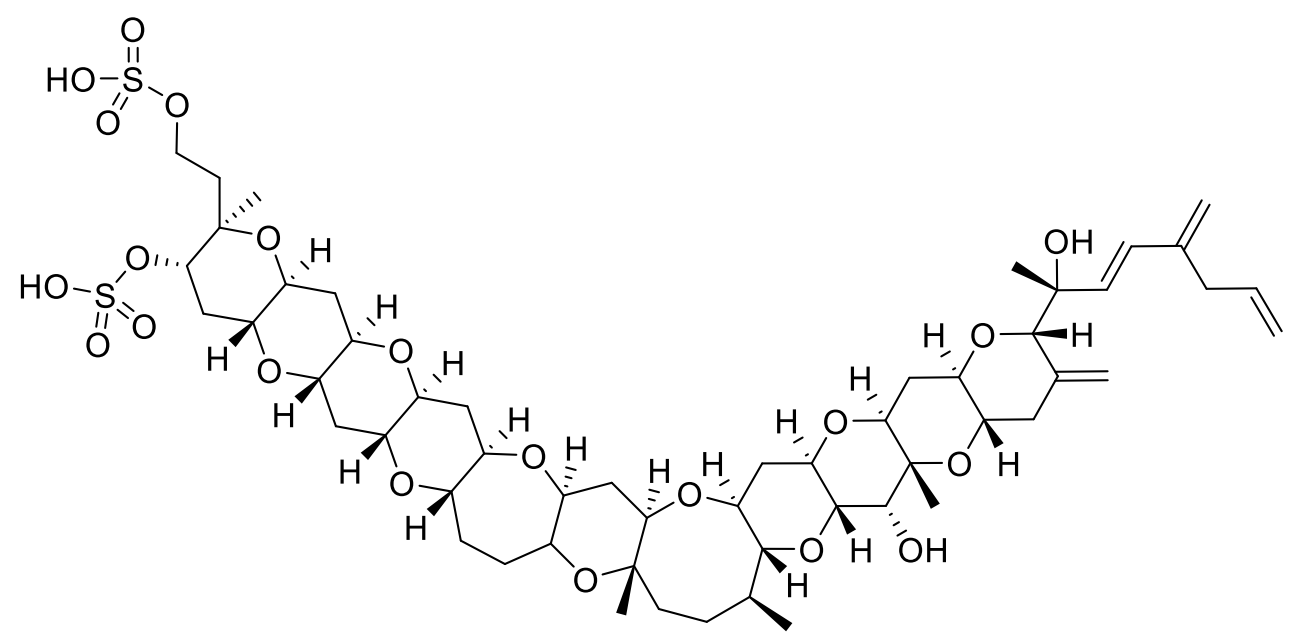

Figure S7. (a) Structure of YTX (1) 


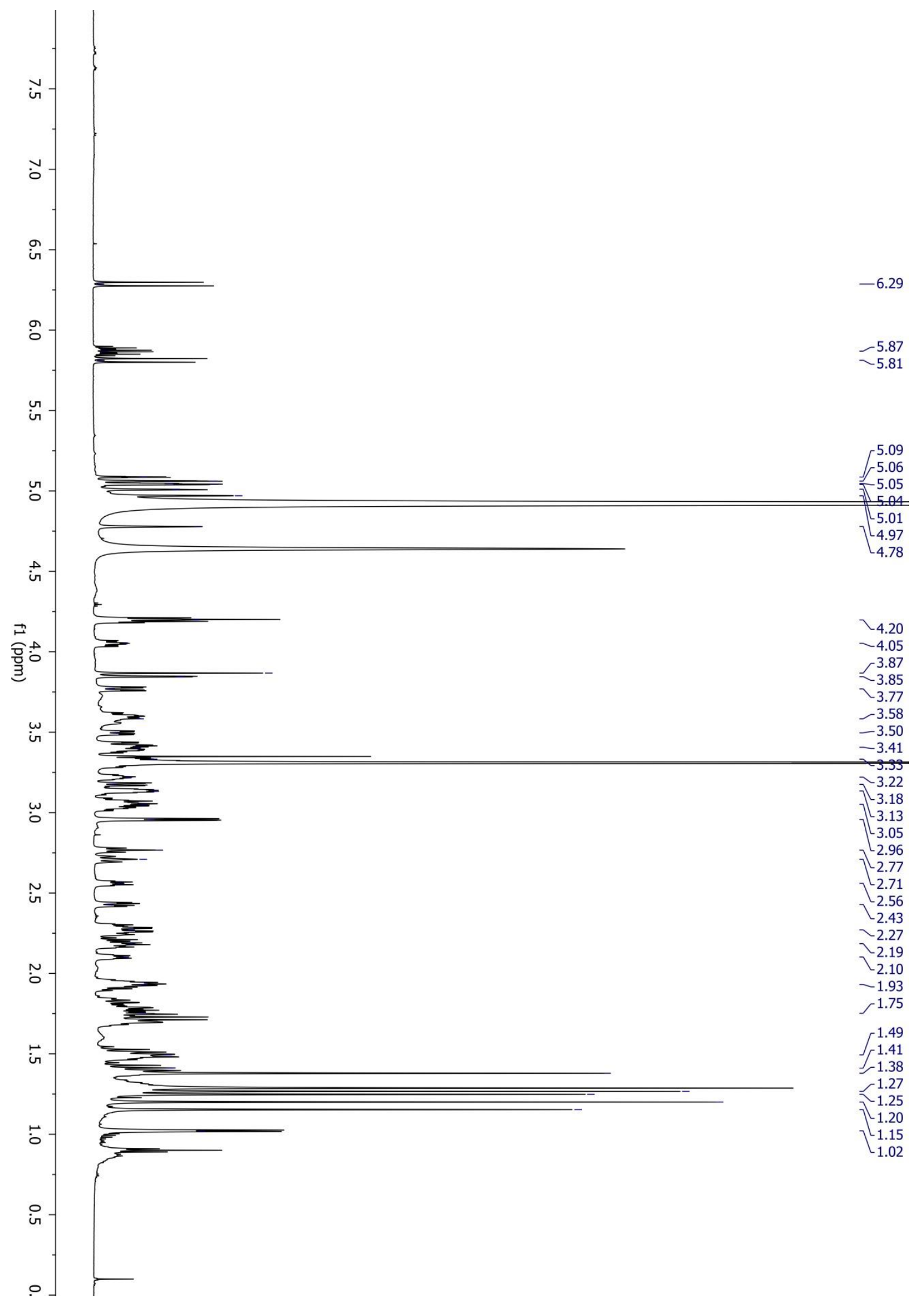

Figure S7. (b) ${ }^{1} \mathrm{H}$ 


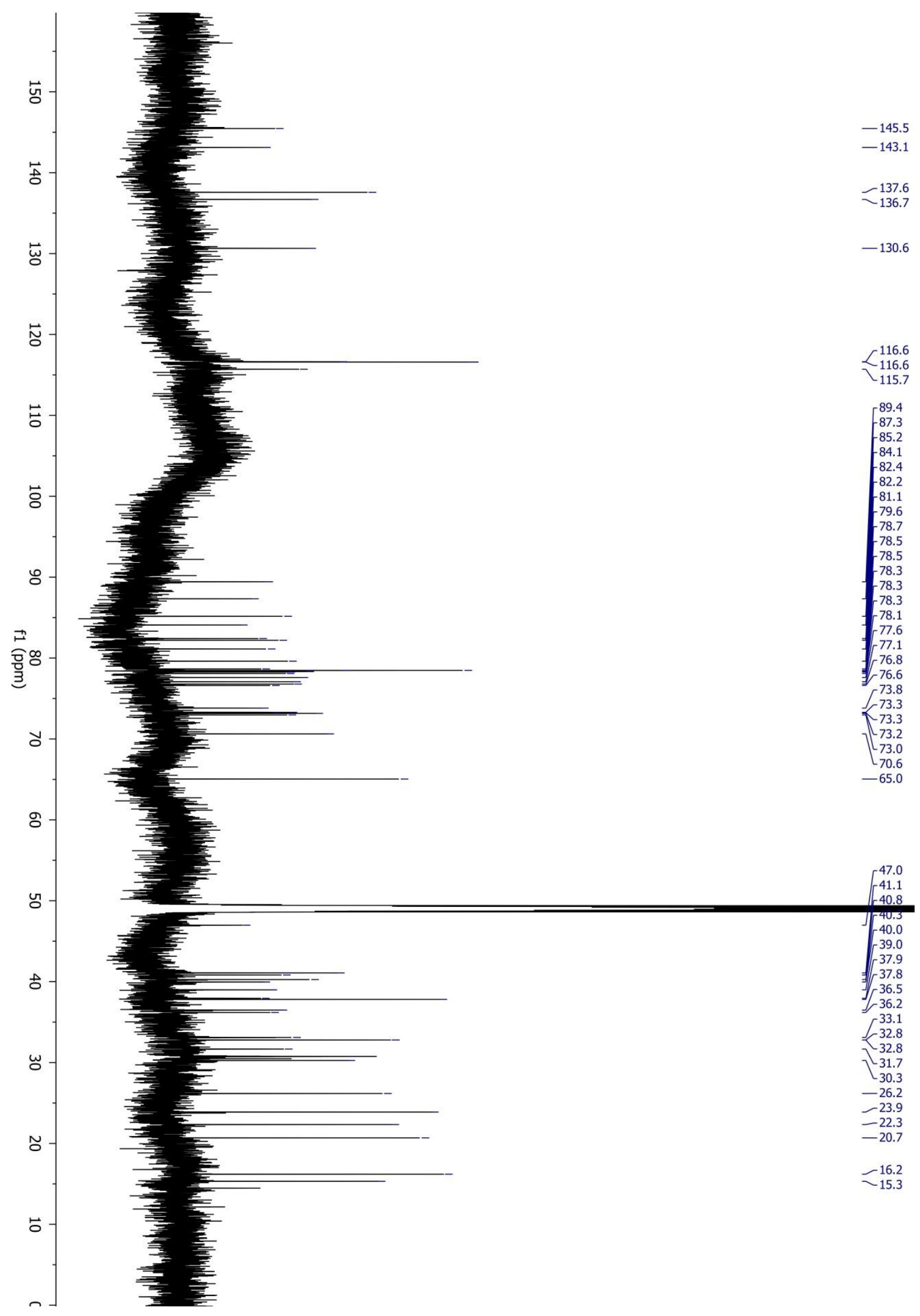

Figure S7. (c) ${ }^{13} \mathrm{C}$ 
YTX

$1141 \rightarrow 1061 \rightarrow 489 \rightarrow 419,407,363$

$1141 \rightarrow 1061 \rightarrow 559$

$1141 \rightarrow 1061 \rightarrow 671 \rightarrow 575,489,349,236$

$1141 \rightarrow 1061 \rightarrow 713 \rightarrow 657$

$1141 \rightarrow 1061 \rightarrow 855 \rightarrow 799,743,713$

$1141 \rightarrow 1061 \rightarrow 924$

24-hydroxyYTX

$$
\begin{aligned}
& 1157 \rightarrow 1077 \rightarrow 559 \rightarrow 419,489 \\
& 1157 \rightarrow 1077 \rightarrow 575 \rightarrow 349,489 \\
& 1157 \rightarrow 1077 \rightarrow 647 \rightarrow 575,407,236 \\
& 1157 \rightarrow 1077 \rightarrow 729 \rightarrow 701,673,575 \\
& 1157 \rightarrow 1077 \rightarrow 759 \rightarrow 741,729,703,649,605,575,559,489 \\
& 1157 \rightarrow 1077 \rightarrow 871,815 \\
& 1157 \rightarrow 1077 \rightarrow 940 \rightarrow 687 \rightarrow 605,575,363
\end{aligned}
$$

Figure S8. Observed LTQ MS fragmentation pathways of YTX (1) and 24-hydroxyYTX (7). 
Table S1. NMR Spectroscopic Data ( $\left.{ }^{1} \mathrm{H} 700 \mathrm{MHz},{ }^{13} \mathrm{C} 175 \mathrm{MHz}, \mathrm{CD}_{3} \mathrm{OD}\right)$ for 24-HydroxyYTX (7) and Yessotoxin (1)

\begin{tabular}{|c|c|c|c|c|}
\hline \multirow[b]{2}{*}{ position } & \multicolumn{2}{|r|}{ Yessotoxin (1) } & \multicolumn{2}{|r|}{ 24-Hydroxyyessotoxin (7) } \\
\hline & $\delta_{\mathrm{C}}^{\mathrm{a}}$, type & $\delta_{\mathrm{H}}{ }^{\mathrm{a}},(J$ in $\mathrm{Hz})$ & $\delta_{\mathrm{C}}$, type & $\delta_{\mathrm{H}},(J$ in $\mathrm{Hz})$ \\
\hline 1 & $65.0, \mathrm{CH}_{2}$ & $4.20, \mathrm{~m}$ & $65.0, \mathrm{CH}_{2}$ & $4.20, \mathrm{~m}$ \\
\hline 2 & $40.3, \mathrm{CH}_{2}$ & $1.91,2.20, \mathrm{~m}$ & $40.3, \mathrm{CH}_{2}$ & $1.91,2.20, \mathrm{~m}$ \\
\hline 3 & $76.6, \mathrm{C}$ & & $76.6, \mathrm{C}$ & \\
\hline 3-Me & $16.2, \mathrm{CH}_{3}$ & $1.27, \mathrm{~s}$ & $16.2, \mathrm{CH}_{3}$ & $1.27, \mathrm{~s}$ \\
\hline 4 & $78.7, \mathrm{CH}$ & $4.20, \mathrm{~m}$ & $78.7, \mathrm{CH}$ & $4.20, \mathrm{~m}$ \\
\hline 5 & $33.1, \mathrm{CH}_{2}$ & $1.72,2.56, \mathrm{dt}(11.9,4.5)$ & $32.8, \mathrm{CH}_{2}$ & $1.72, \mathrm{q}(11.8), 2.56, \mathrm{dt}(11.8,4.5)$ \\
\hline 6 & $78.5, \mathrm{CH}$ & $3.05, \mathrm{~m}$ & $78.5, \mathrm{CH}$ & $3.05, \mathrm{~m}$ \\
\hline 7 & $70.6, \mathrm{CH}$ & $3.32, \mathrm{~m}$ & $70.6, \mathrm{CH}$ & $3.32, \mathrm{~m}$ \\
\hline 8 & $36.5, \mathrm{CH}_{2}$ & $1.39,2.17, \mathrm{~m}$ & $36.5, \mathrm{CH}_{2}$ & $1.39,2.17, \mathrm{~m}$ \\
\hline 9 & $78.3, \mathrm{CH}$ & $3.14, \mathrm{~m}$ & $78.3, \mathrm{CH}$ & $3.13, \mathrm{~m}$ \\
\hline 10 & $78.3, \mathrm{CH}$ & $3.13, \mathrm{~m}$ & $78.3, \mathrm{CH}$ & $3.13, \mathrm{~m}$ \\
\hline 11 & $36.2, \mathrm{CH}_{2}$ & $1.39,2.25, \mathrm{~m}$ & $36.2, \mathrm{CH}_{2}$ & $1.39,2.25, \mathrm{~m}$ \\
\hline 12 & $77.6, \mathrm{CH}$ & $3.03, \mathrm{~m}$ & $77.6, \mathrm{CH}$ & $3.03, \mathrm{~m}$ \\
\hline 13 & $78.1, \mathrm{CH}$ & $3.06, \mathrm{~m}$ & $78.1, \mathrm{CH}$ & $3.06, \mathrm{~m}$ \\
\hline 14 & $37.9, \mathrm{CH}_{2}$ & $1.43,2.29, \mathrm{~m}$ & $38.0, \mathrm{CH}_{2}$ & $1.43,2.29, \mathrm{~m}$ \\
\hline 15 & $81.1, \mathrm{CH}$ & $3.33, \mathrm{~m}$ & $81.1, \mathrm{CH}$ & $3.33, \mathrm{~m}$ \\
\hline 16 & $82.2, \mathrm{CH}$ & $3.22, \mathrm{dt},(8.9,4.3)$ & $82.4, \mathrm{CH}$ & $3.21, \mathrm{~m}$ \\
\hline 17 & $30.3, \mathrm{CH}_{2}$ & $1.79,1.95, \mathrm{~m}$ & $30.2, \mathrm{CH}_{2}$ & $1.79,1.95, \mathrm{~m}$ \\
\hline 18 & $41.1, \mathrm{CH}_{2}$ & $1.78,1.83, \mathrm{~m}$ & $40.8, \mathrm{CH}_{2}$ & $1.83,1.90, \mathrm{~m}$ \\
\hline 19 & $78.5, \mathrm{C}$ & & $78.5, \mathrm{C}$ & \\
\hline 19-Me & $23.9, \mathrm{CH}_{3}$ & $1.25, \mathrm{~s}$ & $23.4, \mathrm{CH}_{3}$ & $1.25, \mathrm{~s}$ \\
\hline 20 & $82.4, \mathrm{CH}$ & $3.43, \mathrm{~m}$ & $82.2, \mathrm{CH}$ & $3.40, \mathrm{~m}$ \\
\hline 21 & $33.1, \mathrm{CH}_{2}$ & $1.73,1.94, \mathrm{~m}$ & $32.5, \mathrm{CH}_{2}$ & $1.78,1.96, \mathrm{~m}$ \\
\hline 22 & $87.3, \mathrm{CH}$ & $3.49, \mathrm{dd}(11.9,4.5)$ & $84.0, \mathrm{CH}$ & $3.39, \mathrm{~m}$ \\
\hline 23 & $77.1, \mathrm{C}$ & & $79.6, \mathrm{C}$ & \\
\hline 23-Me & $20.7, \mathrm{CH}_{3}$ & $1.15, \mathrm{~s}$ & $15.1, \mathrm{CH}_{3}$ & $1.14, \mathrm{~s}$ \\
\hline 24 & $47.0, \mathrm{CH}_{2}$ & $1.48,1.75, \mathrm{~m}$ & $84.2, \mathrm{CH}$ & $3.48, \mathrm{~d}(10.2)$ \\
\hline 25 & $32.8, \mathrm{CH}_{2}$ & $1.48,1.71, \mathrm{~m}$ & $41.5, \mathrm{CH}_{2}$ & $1.39,1.98, \mathrm{~m}$ \\
\hline 26 & $40.8, \mathrm{CH}$ & $1.70, \mathrm{~m}$ & $36.8, \mathrm{CH}$ & $1.82, \mathrm{~m}$ \\
\hline 26-Me & $22.3, \mathrm{CH}_{3}$ & $1.02, \mathrm{~d}(6.2)$ & $22.4, \mathrm{CH}_{3}$ & $1.08, \mathrm{~d}(6.8)$ \\
\hline 27 & $89.4, \mathrm{CH}$ & $2.76, \mathrm{t}(9.1)$ & $89.0, \mathrm{CH}$ & $2.76, \mathrm{t}(9.4)$ \\
\hline 28 & $84.1, \mathrm{CH}$ & $3.30, \mathrm{~m}$ & $83.5, \mathrm{CH}$ & $3.23, \mathrm{~m}$ \\
\hline 29 & $40.0, \mathrm{CH}_{2}$ & $1.52,2.27, \mathrm{~m}$ & $39.7, \mathrm{CH}_{2}$ & $1.50,2.25, \mathrm{~m}$ \\
\hline 30 & $73.3, \mathrm{CH}$ & 3.60, ddd $(11.7,9.8,4.5)$ & $73.3, \mathrm{CH}$ & 3.59, ddd $(4.4,9.8,11.7)$ \\
\hline 31 & $79.6, \mathrm{CH}$ & 3.18, dd $(11.9,4.5)$ & $79.7, \mathrm{CH}$ & $3.19, \mathrm{dd}(9.8,2.9)$ \\
\hline 32 & $73.8, \mathrm{CH}$ & $3.84, \mathrm{~d}(2.8)$ & $73.8, \mathrm{CH}$ & $3.85, \mathrm{~d}(2.7)$ \\
\hline 33 & $76.8, \mathrm{C}$ & & $76.8, \mathrm{C}$ & \\
\hline 33-Me & $15.3, \mathrm{CH}_{3}$ & $1.20, \mathrm{~s}$ & $15.3, \mathrm{CH}_{3}$ & $1.20, \mathrm{~s}$ \\
\hline 34 & $73.3, \mathrm{CH}$ & $3.77, \mathrm{dd}(12.6,3.9)$ & $73.3, \mathrm{CH}$ & $3.76, \mathrm{dd}(12.6,4.0)$ \\
\hline 35 & $31.7, \mathrm{CH}_{2}$ & $1.48, \mathrm{~m}, 2.10, \mathrm{dt}(11.0,4.2)$ & $31.7, \mathrm{CH}_{2}$ & $1.48, \mathrm{~m}, 2.10, \mathrm{dt}(10.9,4.2)$ \\
\hline 36 & $73.2, \mathrm{CH}$ & 4.05, ddd $(11.4,9.5,4.5)$ & $73.2, \mathrm{CH}$ & 4.05, ddd $(11.5,9.6,4.6)$ \\
\hline
\end{tabular}




$\begin{array}{cll}\mathbf{3 7} & 73.0, \mathrm{CH} & 3.39, \mathrm{~m} \\ \mathbf{3 8} & 39.0, \mathrm{CH}_{2} & 2.43, \mathrm{dd}(12.5,4.5), 2.71, \mathrm{td}(13.1,10.6) \\ \mathbf{3 9} & 143.2, \mathrm{C} & \\ \mathbf{3 9 - M e t h y l e n e} & 115.7, \mathrm{CH}_{2} & 4.78, \mathrm{~s}, 5.00, \mathrm{~s} \\ \mathbf{4 0} & 85.2, \mathrm{CH} & 3.87, \mathrm{~s} \\ \mathbf{4 1} & 78.3, \mathrm{C} & \\ \mathbf{4 1 - M e} & 26.2, \mathrm{CH}_{3} & 1.38, \mathrm{~s} \\ \mathbf{4 2} & 136.7, \mathrm{CH} & 5.81, \mathrm{~d}(16.0) \\ \mathbf{4 3} & 130.6, \mathrm{CH} & 6.28, \mathrm{~d}(16.0) \\ \mathbf{4 4} & 145.5, \mathrm{C} & \\ \mathbf{4 4 -} \mathbf{M e t h y l e n e} & 116.6, \mathrm{CH}_{2} & 4.97, \mathrm{~s}, 5.04, \mathrm{~s} \\ \mathbf{4 5} & 37.8, \mathrm{CH}_{2} & 2.96, \mathrm{dd}(6.6,1.5) \\ \mathbf{4 6} & 137.6, \mathrm{CH}^{-} & 5.87, \mathrm{ddt}(16.8,10.1,6.6) \\ \mathbf{4 7} & 116.6, \mathrm{CH}_{2} & 5.06, \mathrm{~m}, 5.08, \mathrm{dd}(1.8,1.7)\end{array}$

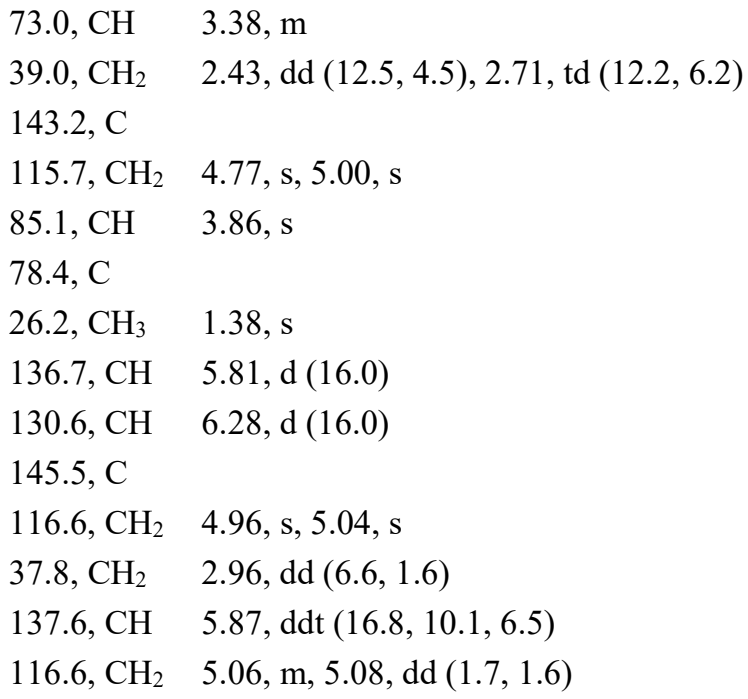

${ }^{a}$ Yessotoxin assignments were acquired on a purified sample of YTX. $\delta_{\mathrm{C} \text { and }} \delta_{\mathrm{H}}$ were comparable to previously reported values. ${ }^{37}$ 